\title{
Particle simulation of Lyapunov exponents in one-component strongly coupled plasmas
}

\author{
Y. Ueshima and K. Nishihara \\ Institute of Laser Engineering, Osaka University, Suita, Osaka 565, Japan \\ D. M. Barnett and T. Tajima \\ Fusion Studies, University of Texas at Austin, Austin, Texas 78712 \\ H. Furukawa \\ Institute for Laser Technology, Suita, Osaka 565, Japan \\ (Received 24 April 1996; revised manuscript received 14 August 1996)
}

\begin{abstract}
The Lyapunov exponents and instantaneous expansion rates in a phase space of Coulomb many-body systems are measured with the use of a three-dimensional particle code SCOPE [K. Nishihara, Kakuyugo Kenkyu 66, 253 (1991)]. The code calculates particle dynamics determined by Coulomb forces among individual particles. The Lyapunov exponents normalized by plasma frequency are found to be proportional to $\Gamma^{-2 / 5}$ in the range of $1 \leqslant \Gamma \leqslant 160$, where $\Gamma$ is the Coulomb coupling constant of the ion one-component plasma. There is a large jump of the Lyapunov exponent near $\Gamma \sim 170$, which corresponds to the phase transition from the liquid to the solid state in the one-component plasma. In the solid state, the normalized Lyapunov exponents are proportional to $\Gamma^{-6 / 5}$ for $170<\Gamma<300$. The observed dependence is discussed in analogy to a rigid-body particle system and a weakly nonlinear lattice system for liquid and solid states, respectively. Diffusion coefficients are found to be proportional to the third power of the Lyapunov exponent in the liquid state, that is, for $1 \leqslant \Gamma \leqslant 160$. These results imply that the Lyapunov exponent is in close relation to the transport processes. The instantaneous expansion rate starts from a small value and increases rapidly to a large peak value before declining slowly towards an asymptotic value. This stage is called the Lyapunov transient stage. Products of the transient time and the Lyapunov exponent are found to be 1.5-2. Information of the initial state is lost after the transient time. The chaotic behavior of the instantaneous expansion rate is also shown. [S1063-651X(97)08003-3]
\end{abstract}

PACS number(s): 52.20.-j, 52.65.-y, 02.50.Ey, 05.70.-a

\section{INTRODUCTION}

A Hamilton system is time reversible. However, in manybody Hamilton systems, the coarse-grained systems may become time irreversible. The coarse-grained phase-space volume increases continuously and covers the whole phase space. The time evolution of the coarse-grained system can then be described macroscopically with small freedom. The ergodicity and reduction of freedom are caused by the sensitivity of a trajectory in the phase space to the initial conditions, in other words, the trajectory instability in the phase space. The loss of initial state information must be related to transport processes, which are macroscopically time irreversible. The Lyapunov exponent determines the growth rates of the trajectory instability, namely, the rates at which the initial-state information is lost. The Lyapunov exponents in the phase space are mechanical characteristic quantities and they are definable even for strong nonequilibrium in which statistical quantities are hard to calculate or have no meaning. The Lyapunov exponent can be useful in studying the microscopic evolution and macroscopic description of the systems. Recently, there has been a great deal of research effort devoted to finding the relationship between the Lyapunov exponents and macroscopic statistical quantities [1-9]. In previous works [1-7], only the systems that are determined by short-range forces were considered. In this paper, we consider a Coulomb many-body system whose dynamics is determined by long-range forces.
In a high-temperature and low-density plasma, correlations among particles can be ignored and the plasma can be treated as an ideal plasma. In the ideal plasma, a test charge is shielded effectively at a distance of the order of the Debye length

$$
\lambda_{D}=\sqrt{\frac{4 \pi n e^{2}}{T}}
$$

and the number of particles in a Debye sphere $N_{D}$ is large,

$$
N_{D} \equiv \frac{4 \pi}{3} n \lambda_{D}^{3} \gg 1
$$

where $e, T$, and $n$ are the charge, temperature in energy units, and number density, respectively. Transport processes are determined mainly by macroscopic fields and may not be accompanied by the loss of the initial-state information. On the other hand, in a dense plasma, the correlations among particles cannot be ignored. The Debye length is not an effective shielding length because the number of particles in the Debye sphere is small. The plasmas are called strongly coupled plasmas and the direct interaction among particles and microscopic fields dominates transport processes that are accompanied by the loss of initial-state information. The one-component plasma is chosen as the object of study because it is simple and a good approximation for dense plas- 
mas. Ions are point charges and the electrons can be regarded as a uniform background. Ion one-component plasmas are characterized by only one nondimensional parameter, i.e., the Coulomb coupling constant

$$
\Gamma \equiv \frac{e^{2}}{a T}, \quad \frac{4 \pi}{3} a^{3} \equiv \frac{1}{n},
$$

where $a$ is the ion sphere radius. The Coulomb coupling constant represents the ratio of the Coulomb interaction energy between two particles, separated a distance equal to the ion sphere radius, to the mean kinetic energy of a particle. The relation between the Coulomb coupling constant and the number of particles in a Debye sphere is given by $N_{D}=(3 \Gamma)^{-3 / 2}$. If the Coulomb coupling constant is much smaller than one, the plasma can be regarded as an ideal plasma. On the other hand, if the Coulomb coupling constant is larger than one, the plasma must be regarded as a dense plasma. It has short-range order and behaves like a liquid. The one-component dense plasma has the phase transition from the liquid to the solid state at $\Gamma \sim 170$ [10-13]. As shown later, these properties are confirmed by simulations.

The separation between two adjacent trajectories in the $6 N$ phase space is calculated for a long time. It will be shown that the separation expands exponentially in time and saturates at certain distances in the momentum and position coordinates spaces. By imposing the rescaling method [1417] to prevent the saturation, the instantaneous expansion rate is observed for a long time and the Lyapunov exponent is obtained as its time-averaged value.

The instantaneous expansion rate starts from a small value and increases rapidly to a large peak value before declining slowly towards an asymptotic value $[1,8]$. This stage is called the Lyapunov transient stage. Evans, Cohen, and Morriss [1] have derived a short-time formula for the instantaneous expansion rates in the transient stage. In this paper, the short-time behavior of the instantaneous expansion rates is also investigated and products of the transient time and the Lyapunov exponent are found to be 1.5-2. Information of the initial state is almost lost within the transient time. Amitrano and Berry [2] evaluated the probability distribution of the instantaneous expansion rates for a classical $\mathrm{Ar}^{3}$ cluster. They found that the probability distribution of the instantaneous expansion rates is in close relation to the ergodicity of the system. We observe chaotic behaviors and universal spectra of the instantaneous expansion rates.

In previous papers [3-5], many analytical formulas of the Lyapunov exponent were proposed. Pettini and Landolfi [4] calculated the Lyapunov exponents in a nonlinear lattice for various energy densities and found the relation between the Lyapunov exponent and the energy density. In this paper, the dependence of the Lyapunov exponent on the Coulomb coupling constant is investigated in detail for $1<\Gamma<300$. The dependence is discussed for the liquid state $\Gamma<170$ and the solid state $\Gamma>170$ separately.

Many authors [6-9] conjecture that the Lyapunov exponents must be related to the macroscopic transport coefficients because they are connected to the trajectory instability in the phase space, which involves the loss of initial-state information. Gaspard and Nicolis [6] found a connection between the Lyapunov exponent and the diffusion coefficient of a two-dimensional Lorentz gas. Posch and Hoover [7] calculated the Lyapunov exponents of a dense Lennard-Jones particle system and found a connection between the Lyapunov exponent and the color conductivity in a nonequilibrium molecular-dynamics simulation. We present here a relation between the Lyapunov exponents and the diffusion coefficients for ion one-component strongly coupled plasmas.

In Sec. II we show the numerical method to calculate the Lyapunov exponent and instantaneous expansion rate with the three-dimensional strongly coupled plasma particle code (SCOPE) [18,19]. In Sec. III the results obtained with the nonrescaled method are discussed, such as the saturation of the separation between two nearby trajectories. In Sec. IV the dependence of the Lyapunov exponent on the Coulomb coupling constant is investigated in detail for $1<\Gamma<300$. The dependence is also discussed for the liquid state $(\Gamma<170)$ and the solid state $(\Gamma>170)$ separately.

\section{LYAPUNOV EXPONENT AND INSTANTANEOUS EXPANSION RATE}

\section{A. Definition of the instantaneous expansion rate}

A classical three-dimensional system of $N$ particles has $3 N$ momentum $\mathbf{p}$ and $3 N$ position coordinates $\mathbf{q}$. Therefore, $\Lambda(\mathbf{p}, \mathbf{q})$ represents a $6 N$-dimensional phase-space point. We can describe the motions of the particles in the system with the Hamiltonian $H$ as

$$
\begin{gathered}
\dot{\Lambda} \equiv\left(\frac{\dot{\mathbf{p}}}{\dot{\mathbf{q}}}\right)=\left(\begin{array}{c}
-\frac{\partial H}{\partial \mathbf{q}} \\
\frac{\partial H}{\partial \mathbf{p}}
\end{array}\right) \equiv \mathbf{G}(\Lambda), \\
H=\frac{1}{2} \sum m \mathbf{v}^{2}+\Phi,
\end{gathered}
$$

where $m, \mathbf{v}$, and $\Phi$ represent mass, velocity, and potential energy. By assuming that particles have the same mass and the potential-energy depends only on the $3 N$ position coordinates, we obtain

$$
\frac{\partial H}{\partial \mathbf{q}}=\frac{\partial \Phi(\mathbf{q})}{\partial \mathbf{q}}, \quad \frac{\partial H}{\partial \mathbf{p}}=\frac{\mathbf{p}}{m}
$$

Equation (2.1) gives a trajectory of the $6 N$-dimensional phase-space point $\Lambda(t)$.

The Lyapunov exponent is an index of the trajectory instability in the $6 N$ phase space. We consider a small perturbation of the trajectory in the $6 N$ phase space to obtain the Lyapunov exponent. This perturbation can be regarded as a displacement that represents the difference between a reference point $\boldsymbol{\Lambda}_{r}(t)$ and a displaced point $\boldsymbol{\Lambda}_{d}(t)$ in the phase space. Namely, the displacement is defined as $\boldsymbol{\delta}(t)=\boldsymbol{\Lambda}_{d}(t)-\boldsymbol{\Lambda}_{r}(t)$. Both the reference and displaced trajectories are the solutions of Eq. (2.1). An equation of motion for the displacement is obtained by linearizing Eq. (2.1), 


$$
\begin{gathered}
\dot{\boldsymbol{\delta}} \equiv\left(\begin{array}{c}
\delta \dot{\mathbf{p}} \\
\dot{\delta} \mathbf{q}
\end{array}\right)=T(\boldsymbol{\Lambda}) \boldsymbol{\delta}, \\
T(\boldsymbol{\Lambda}) \equiv \frac{\partial \mathbf{G}(\boldsymbol{\Lambda})}{\partial \boldsymbol{\Lambda}}=\left(\begin{array}{cc}
0 & -\frac{\partial \Phi(\mathbf{q}(t))}{\partial \mathbf{q} \partial \mathbf{q}} \\
1 & 0
\end{array}\right) .
\end{gathered}
$$

This equation can be solved formally as

$$
\begin{gathered}
\boldsymbol{\delta}(t)=X(t) \boldsymbol{\delta}(0), \\
X(t) \equiv \exp \left(\int_{0}^{t} d \tau T(\boldsymbol{\Lambda}(\tau))\right) .
\end{gathered}
$$

The Lyapunov exponent represents the averaged exponential rate of the divergence of the displacement. It depends on an initial reference point and the direction of the displacement in the phase space

$$
\begin{aligned}
\lambda(\Lambda(0), \mathbf{e}) & \equiv \lim _{t \rightarrow \infty} \frac{1}{t} \ln \left[\frac{\|X(t) \mathbf{e}\|}{\|\mathbf{e}\|},\right. \\
\mathbf{e} & \equiv \frac{\boldsymbol{\delta}(0)}{\|\boldsymbol{\delta}(0)\|} .
\end{aligned}
$$

A new variable can be defined as an instantaneous divergence of two initially close trajectories by

$$
\lambda_{\text {inst }}(\mathbf{\Lambda}(t), \mathbf{e}) \equiv \frac{\|X \dot{(t)} \mathbf{e}\|}{\|X(t) \mathbf{e}\|},
$$

which is called the instantaneous expansion rate. A time variation of the instantaneous expansion rate informs us about the uniformity of the phase mixing and microscopic process in the phase space in detail. In addition, the Lyapunov exponent is redefined as a time-averaged value of the instantaneous expansion rate

$$
\lambda=\left\langle\lambda_{\text {inst }}\right\rangle \equiv \lim _{t \rightarrow \infty} \frac{1}{t} \int_{0}^{t} d t \lambda_{\text {inst }}(\mathbf{\Lambda}(t), \mathbf{e}) .
$$

If the system is ergodic, the Lyapunov exponent depends not on the initial point $\boldsymbol{\Lambda}(0)$, but only on the direction of the initial displacement $\mathbf{e}$ in the phase space. A $6 \mathrm{~N}$-dimensional vector e can be expressed as a linear combination of the $6 \mathrm{~N}$ base vectors. In principle, the Lyapunov exponent given by Eq. (2.7) coincides with one of the $6 \mathrm{~N}$ Lyapunov exponents corresponding to the $6 \mathrm{~N}$ bases of the eigenvectors. If the direction of the initial displacement is chosen at random ("random" means that the direction is not perpendicular to any base eigenvectors), it has a component of the base eigenvector corresponding to the maximum Lyapunov exponent.

\section{B. Numerical calculation method}

If the trajectory $\boldsymbol{\Lambda}(t)$ depends sensitively on the initial conditions, the displaced trajectory separates exponentially in time from the reference trajectory. The Lyapunov exponent defined by Eq. (2.5) can be calculated by

$$
\lambda=\frac{1}{t} \ln \left(\frac{|\boldsymbol{\delta}(t)|}{|\boldsymbol{\delta}(0)|}\right)
$$

for large time $t$ and small initial displacement $|\boldsymbol{\delta}(0)|$.

In the numerical calculation, we must determine an appropriate amplitude of the initial displacement so that the displacement can be treated as a perturbation of the reference trajectory and it does not cause numerical error in the calculation of the Lyapunov exponent. The initial separation between two trajectories cannot be set equal to a very small value because an excessively small separation causes large numerical errors. Even if the initial separation is small enough, because of its exponential growth, the separation becomes too large in a short time to observe the Lyapunov exponent. To avoid this difficulty the amplitude of the displacement should be rescaled. It should be noted here that the Lyapunov exponent depends not on the amplitude of the displacement, but on its direction.

The displacement is rescaled as follows [14-17]. In the numerical calculation, the reference and displaced trajectories $\boldsymbol{\Lambda}_{r}(t)$ and $\boldsymbol{\Lambda}_{d}(t)$ are defined only at a discrete time with a finite time increment $\Delta t$. At the $n$th time step, the $n$th rescaling factor is calculated as

$$
f(n)_{\text {scale }}=\frac{|\boldsymbol{\delta}((n-1) \Delta t)|}{|\boldsymbol{\delta}(n \Delta t)|} .
$$

A new starting point, $\boldsymbol{\Lambda}_{d}^{\prime}(n \Delta t)$ of the displaced trajectory at the $n$th time step is then determined by

$$
\boldsymbol{\Lambda}_{d}^{\prime}(n \Delta t)=\boldsymbol{\Lambda}_{r}(n \Delta t)+f(n)_{\text {scale }} \boldsymbol{\delta}(n \Delta t) .
$$

The instantaneous expansion rate can be calculated as

$$
\lambda_{\text {inst }}(n \Delta t)=\frac{1}{\Delta t} \ln \left(\frac{|\boldsymbol{\delta}(n \Delta t)|}{|\boldsymbol{\delta}(0)|}\right) .
$$

The rescaling procedure makes long-time calculation possible. During that time, an initial direction of the displacement turns to the maximum stretching direction corresponding to the largest Lyapunov exponent. We obtain the largest Lyapunov exponent in the system as

$$
\lambda \equiv \lim _{n_{\max } \rightarrow \infty} \frac{1}{n_{\max } \Delta t} \sum_{n=1}^{n_{\max }} \lambda_{\text {inst }}(n \Delta t) .
$$

\section{Simulation code}

We perform $N V E$-invariant molecular-dynamics simulation with the use of a three-dimensional particle code SCOPE $[18,19]$, where $N, V$, and $E$ represent the number of particles, volume, and total energy. It is necessary for particle simulations of dense plasmas to calculate precisely the Coulomb interactions among close and distant particles. It is, however, very expensive to calculate forces among all particles. The particle-particle-particle-mesh (PPPM) method [18-20] is used in the code to treat many particles, in which particleparticle method is employed for the calculation of the forces among close particles and the particle-mesh method [21] is employed for the calculation of the forces among distant 


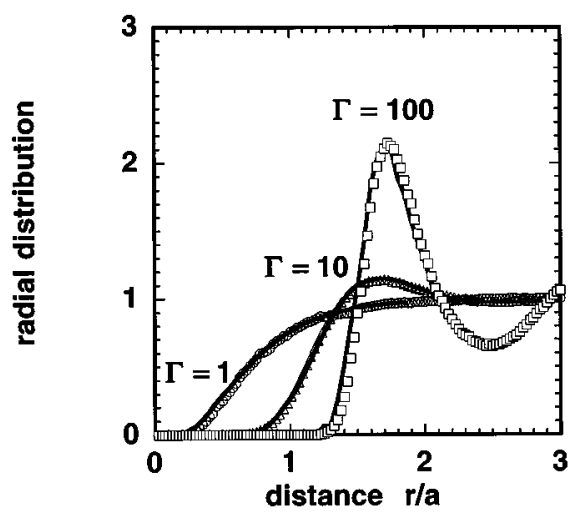

FIG. 1. Radial distribution functions obtained by SCOPE. Horizontal axis is the distance normalized by the ion sphere radius. Open circles, triangle, and square represent present simulation results for $\Gamma=1,10$, and 100 , and lines represent Monte-Carlo [10 12] simulation results.

particles. The Coulomb forces among close particles are directly summed up in the particle-particle method, while in the particle-mesh method the forces on a particles are interpolated from electric fields of mesh points. The electric fields are obtained by solving the Poisson equation with charges assigned on the mesh points. To reduce numerical errors caused by the particle-mesh method, the original PPPM method developed by Hockney and Eastwood [20] is improved in SCOPE [18]. SCOPE is described in detail Appendix A.

Our system comprises 500 ions in a unit cubic box and the periodic boundary condition is adopted. The Coulomb force on a particle is directly summed up over approximately 210 particles. The number of meshes for the particle-mesh method is set equal to $8^{3}=512$. The unit time steps used are $\Delta t \omega_{p}=0.006$ for $\Gamma=1, \Delta t \omega_{p}=0.01$ for $\Gamma=2$, and $\Delta t \omega_{p}=0.02$ for $\Gamma=5-300$, where $\omega_{p}$ is the plasma frequency. To obtain a thermal equilibrium state, we have performed a pre-simulation for a duration of $200 \omega_{p} t$ before the observation of the Lyapunov exponent. The radial distribution functions are obtained for five periods of plasma oscillation in the presimulation and their time-averaged values are shown in Fig. 1. They agree quite well with Monte Carlo simulation results [10-13], which indicates the validity of SCOPE.

\section{TIME VARIATION OF DISPLACEMENT}

\section{A. Exponential divergence of nearby trajectories}

Before calculating the Lyapunov exponent with the continuous rescaled method, we confirm that the displacement diverges exponentially in time. The initial displacement should be an appropriate amplitude, as we have discussed in Sec. II. We perform time-advanced calculations of the separation between reference and displaced systems with the nonrescaled method for various Coulomb coupling constants. As a result of the calculations, we will show that the nearby trajectories separate exponentially in time.

In the six-dimensional space $\left(p_{x}, p_{y}, p_{z}, q_{x}, q_{y}, q_{z}\right)$, the magnitude of the initial displacement is given by the normal distribution and its direction is isotropic. The root mean squares (rms's) of the displacements are set to be
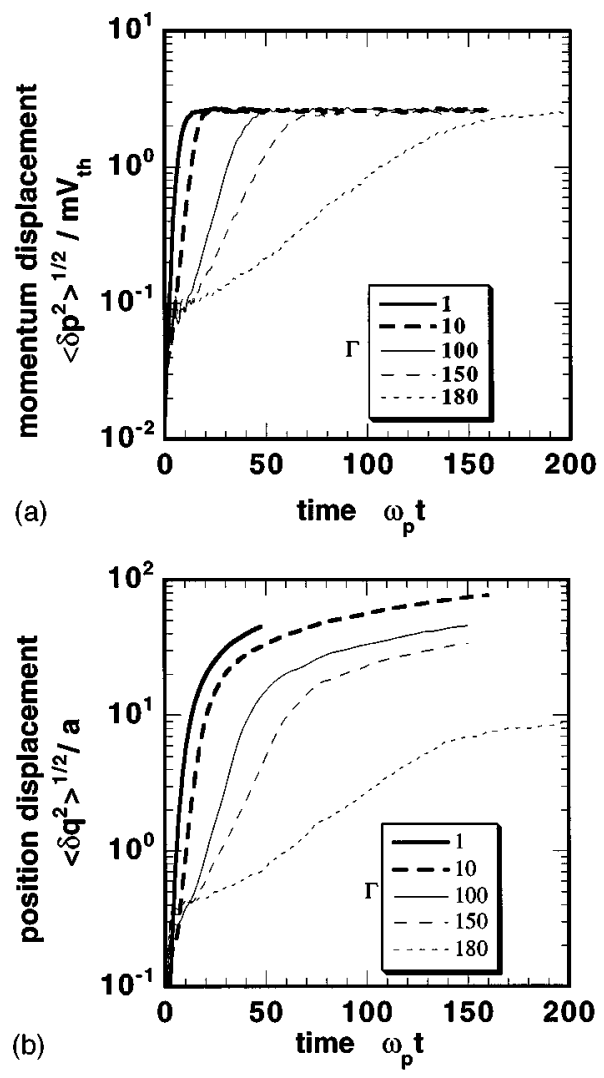

FIG. 2. Time evolution of RMS separation distances between nearby trajectories in the momentum space (a) and position coordinates space (b) for various Coulomb coupling constants $\Gamma=1,10$, 100, 150, and 180. Time, momentum, and position are normalized by plasma frequency $\omega_{p}$, product of mass, and thermal speed $m v_{\text {th }}$, and ion sphere radius $a$, respectively.

$5.0 \times 10^{-3} m v_{\text {th }}$ in the momentum space and $5.0 \times 10^{-3} a$ in the position coordinates space, where $v_{\text {th }}$ is thermal speed $m v_{\text {th }}^{2}=T$. These values $5.0 \times 10^{-3} m v_{\text {th }}$ and $5.0 \times 10^{-3} a$ result in a high enough accuracy for calculating the divergence of the nearby trajectories. Figures 2(a) and 2(b) show the time variations of the rms displacements in the $3 \mathrm{~N}$-dimensional momentum and position coordinates spaces. The rms displacement starts from small values and varies irregularly in time before starting to diverge exponentially. This irregular transient stage is called the Lyapunov transient stage. In the transient stage, the initial displacement seeks out the eigendirection in the phase space corresponding to the largest eigenvalue, which is independent of time and the direction of the initial displacement. We will discuss this transient stage in detail in Sec. IV. After the Lyapunov transient stage, it is clearly seen that the displacements diverge exponentially in time for the various Coulomb coupling constants. This result indicates that the chosen initial displacement is so small as to be regarded as an infinitesimal value. In other words, the motion of the displacement can be described by the linearized equation (2.3) in this range of the displacement. The expansion rates in the momentum and position coordinates spaces are almost the same values. The exponential growth of the displacements indicates that the system has the positive Lyapunov exponent. The averaged expansion rates decrease with the increase of the Coulomb coupling constant. As shown later, the expansion rates measured by both the 
nonrescaled and rescaled methods are almost the same.

\section{B. Saturation of displacement}

As shown in Figs. 2(a) and 2(b), when the displacements reach certain amplitudes, the diverging speeds deviate from the exponential growth. The deviations from the exponential (linear) growth occur around the separations of $|\delta \mathbf{p}| \sim m v_{\text {th }}$ and $|\delta \mathbf{q}| \sim 3 \sim 4 \lambda_{D}$ for the various Coulomb coupling constants, where $\lambda_{D}$ is $a / \sqrt{3 \Gamma}$. It is interesting to note that the separation $|\delta \mathbf{q}|$ corresponding to the deviation point is determined by the Debye length even for the strongly coupled plasmas.

Following the separation distance for a long time, the rms of the separation distance in the momentum space saturates at $|\delta \mathbf{p}| \sim \sqrt{6} m v_{\text {th }}$ and the one in the position coordinates space diverges as $|\delta \mathbf{q}| \sim t^{1 / 2}$ for the various Coulomb coupling constants. As shown below, these asymptotic behaviors of the displacements indicate that the reference and displaced trajectories are no longer correlated. Assuming that the velocity distributions of both the reference and displaced systems are Maxwellians $f_{M}(p)$ with the same temperature, the displacement in the momentum space is written as

$$
\begin{aligned}
\overline{\delta \mathbf{p}^{2}} & \equiv \frac{1}{N} \sum_{i=1}^{N}\left(\mathbf{p}_{d_{i}}-\mathbf{p}_{r_{i}}\right)^{2}=\frac{1}{N} \sum_{i=1}^{N}\left(\mathbf{p}_{d_{i}}^{2}-2 \mathbf{p}_{r_{i}} \cdot \mathbf{p}_{d_{i}}+\mathbf{p}_{r_{i}}^{2}\right) \\
& =6 m V_{\mathrm{th}}^{2}-\frac{2}{N} \sum_{i=1}^{N} \mathbf{p}_{r_{i}} \cdot \mathbf{p}_{d_{i}},
\end{aligned}
$$

where the relation $(1 / N) \sum_{i}^{N} \mathbf{p}_{i}^{2}=\int_{0}^{\infty} \mathbf{p}^{2} f_{M}(p) d p=3 m V_{\text {th }}$ has been used. The subscripts $r$ and $d$ denote, respectively, reference and displaced systems, and the subscript $i$ represents the $i$ th ion in the corresponding systems. Since the given initial displacement in the momentum space is very small in comparison to $m v_{\text {th }}$, the $3 N$-dimensional momentum vector in the reference system is almost parallel to that in the displaced system. As the rms displacement in the momentum space diverges exponentially, the initial-state information becomes lost. When the initial-state information is perfectly lost, namely, the two systems are not correlated with each other, an angle between the three-dimensional momentum vectors in the reference and displaced systems becomes random. This indicates that the $3 \mathrm{~N}$-dimensional momentum vector in the reference system is almost orthogonal to that in the displaced system. Then the momentum displacement becomes $\sqrt{6} m v_{\text {th }}$.

The displacement in the position coordinates space is written as

$$
\begin{aligned}
\overline{\delta \mathbf{q}^{2}} \equiv & \frac{1}{N} \sum_{i=1}^{N}\left(\mathbf{q}_{d_{i}}-\mathbf{q}_{r_{i}}\right)^{2} \\
= & \frac{1}{N} \sum_{i=1}^{N}\left(d \mathbf{q}_{d_{i}}^{2}+d \mathbf{q}_{r_{i}}^{2}\right)+\frac{1}{N} \sum_{i=1}^{N} 2\left\{-d \mathbf{q}_{d_{i}} \cdot d \mathbf{q}_{r_{i}}\right. \\
& \left.+\left[\mathbf{q}_{d_{i}}(0)-\mathbf{q}_{r_{i}}(0)\right] \cdot\left(d \mathbf{q}_{d_{i}}-d \mathbf{q}_{r_{i}}\right)\right\}+\overline{\delta \mathbf{q}(0)^{2}},
\end{aligned}
$$

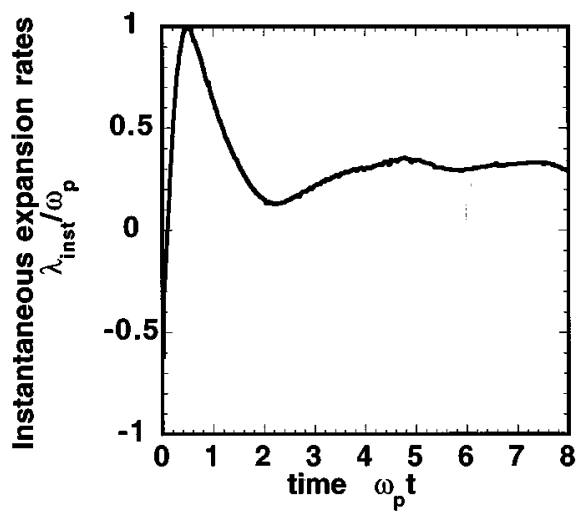

FIG. 3. Instantaneous expansion rate normalized by plasma frequency as a function of time for $\Gamma=10$ in the transient stage.

where $\mathbf{q}_{i}(t) \equiv \mathbf{q}_{i}(0)+d \mathbf{q}_{i}(t)$. When the two systems are not correlated with each other, the first term $\left(d \mathbf{q}_{d_{i}}^{2}+d \mathbf{q}_{r_{i}}^{2}\right)$ in Eq. (3.2) becomes the leading term of the displacement in the position coordinates space. The motion of the displacement is then regarded as a random-walk process. The mean square of the displacement in the position coordinates space is just twice as large as the mean-square deviation of particles in the three-dimensional position coordinates space. As a result, the rms of the displacement in the position coordinate space diverges proportionally to $t^{1 / 2}$ in the liquid state, while it becomes a constant in the solid state.

\section{INSTANTANEOUS EXPANSION RATE}

\section{A. Transient behavior of the instantaneous expansion rate}

To estimate the Lyapunov exponent more accurately, we calculate the instantaneous expansion rates with the rescaled method for the various Coulomb coupling constants. As shown in the preceding section, we should choose the magnitudes of the initial displacement between $5.0 \times 10^{-3} \sim 1 m v_{\text {th }}$ and $5.0 \times 10^{-3} \sim 4 a / \sqrt{3 \Gamma}$ in the momentum and position coordinates spaces, respectively. In the calculation with the rescaling method, the normal distribution with the rms displacements of $5.0 \times 10^{-3} m v_{\text {th }}$ and $5.0 \times 10^{-3} a$ is used in the momentum and position coordinates spaces, respectively. The instantaneous expansion rates give not only the Lyapunov exponent, but also more detailed information about the uniformity of the trajectory instability.

Figure 3 shows the instantaneous expansion rate for $\Gamma=10$. Typically, it starts from a small value and increases rapidly to a large peak value before declining slowly towards an asymptotic value. This stage is called the Lyapunov transient stage. In the transient stage, the initial displacement seeks out the phase-space eigendirection corresponding to the maximum eigenvalue, i.e., the largest Lyapunov exponent. We observe how the displacement varies in the transient stage. As an example, Fig. 4 shows the case that the initial distribution of each component of the displacements $\left(\delta p_{x i}, \delta p_{y i}, \delta p_{z i}, \delta q_{x i}, \delta q_{y i}, \delta q_{z i}\right.$, where $\left.i=1-N\right)$ has two peaks at $\pm 5.0 / \sqrt{3} \times 10^{-3} \mathrm{mv}_{\text {th }}$ in the momentum spaces and $\pm 5.0 / \sqrt{3} \times 10^{-3} a$ in the position coordinates spaces. The distribution relaxes to the normal distribution during the Lyapunov transient time. After the transient time, the distribution does not change for any initial displacements except 


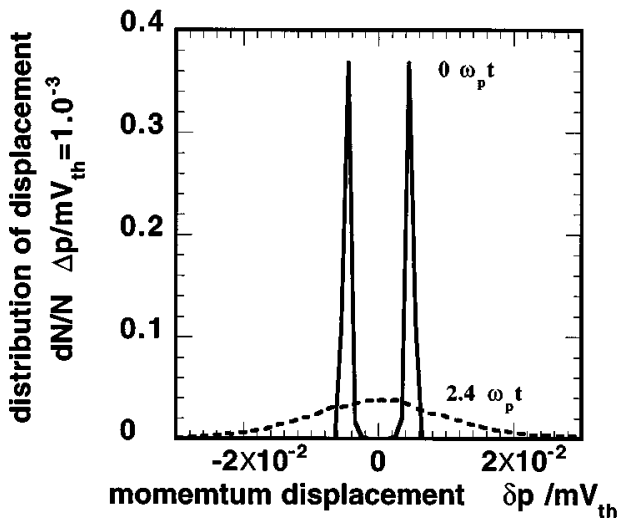

FIG. 4. Distribution functions of the displacement in the momentum space at time $=0$ (solid line) and time $=2.4 \omega_{p} t$ (dashed line) for $\Gamma=10$, where $d N$ represents number of particles of which momentum is $p \sim p+\Delta p, \Delta p / m v_{\mathrm{th}}=10^{-3}$.

for small fluctuations. This result indicates that the initialstate information is lost within the Lyapunov transient time.

The normalized ratio of the displacement magnitude in the momentum to that in the position $|\delta \mathbf{p}| /\left(|\delta \mathbf{q}| \omega_{p}\right)$ also becomes independent of time and the direction of the initial displacement after the transient time. As shown in Fig. 5, the normalized ratio is proportional to $\Gamma^{-1 / 3}$ for $1 \leqslant \Gamma \leqslant 170$ and does not depend on the Coulomb coupling constant for $\Gamma>170$. In Appendix B it is qualitatively explained that the ratio is independent of the Coulomb coupling constant in the solid state with the use of a simple model. The ratio gives part of the information for the eigendirection corresponding to the largest Lyapunov exponent. The result is evidence that any of the directions of displacements turn to the direction corresponding to the largest Lyapunov exponent within the transient time.

Figure 6 shows the time variation of the instantaneous expansion rates for $\Gamma=1,10,50$, and 200. The transient time increases as the Coulomb coupling constant increases, in other words, as the Lyapunov exponent decreases. Figure 7 shows the products of the Lyapunov exponents and the Lyapunov transient times for $\Gamma=1-150$. The products are nondimensional values. As shown in Fig. 7, the products are almost constant of 1.5-2.0, namely, the Lyapunov transient time is proportional to the inverse of the Lyapunov exponent.

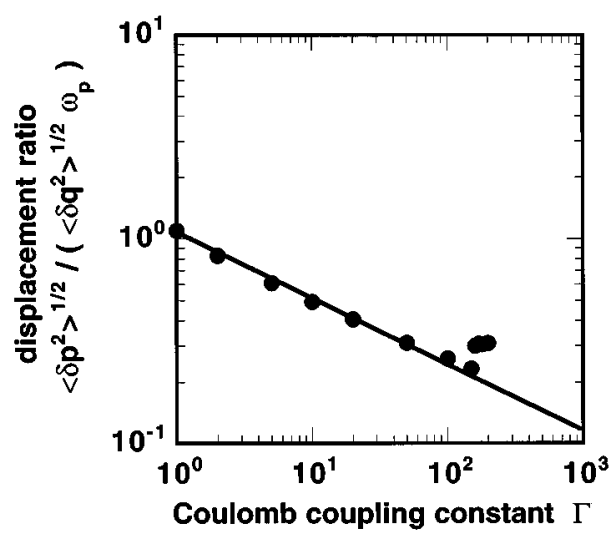

FIG. 5. The ratio $\delta p /\left(\delta q \omega_{p}\right)$ as a function of $\Gamma=1 \sim 200$. A solid line is obtained by a least squares method for $1 \leqslant \Gamma \leqslant 160$.

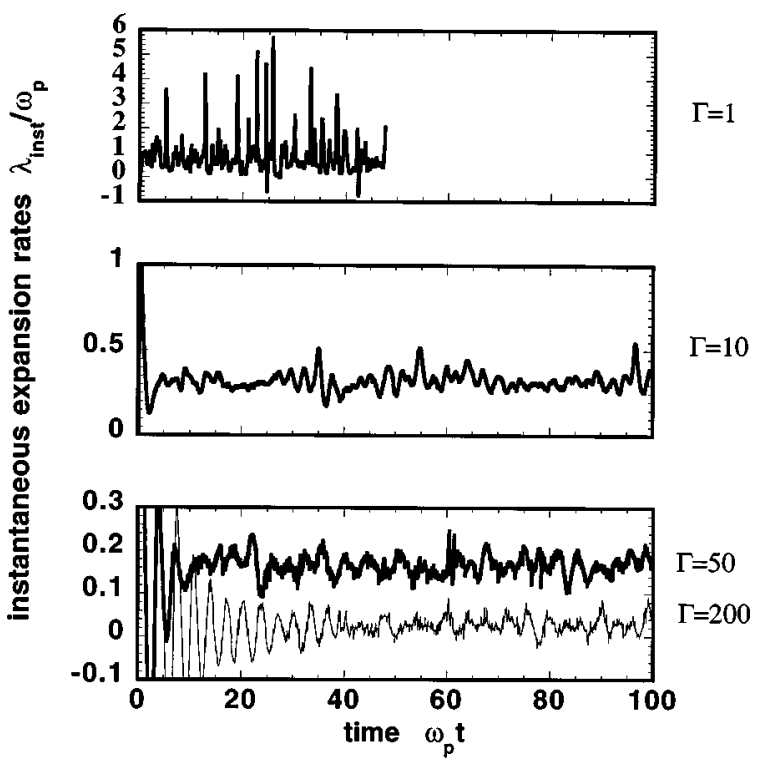

FIG. 6. Normalized instantaneous expansion rates as a function of time for $\Gamma=1,10,50$, and 200 .

\section{B. Spectra of the instantaneous expansion rate}

As shown in Fig. 6, the instantaneous expansion rates have large fluctuations and are chaotic in time. Bursts of the instantaneous expansion rate, which are much larger than its average, are observed for $\Gamma=1$. This result suggests that collisions among particles occur rarely and strongly in a local region. Even for $\Gamma>1$, the fluctuations of the instantaneous expansion rate are as large as their average. These behaviors indicate that the trajectory instability is nonuniform in the phase space. The instantaneous expansion rate in the asymptotic stage may provide the uniformity of the phase mixing and more precise information about the microscopic process. Figure 8 shows the spectra of the instantaneous expansion rates for $\Gamma=1,2,10,50$, and 200. The instantaneous expansion rates have broad spectra and consist of three different spectra. In order of frequency, the first component is a plateau around the plasma frequency, the second decays like $f^{-2}$, and the last decays as $f^{-1}$. The plateau around the plasma frequency may correspond to the collective motion in the plasma.

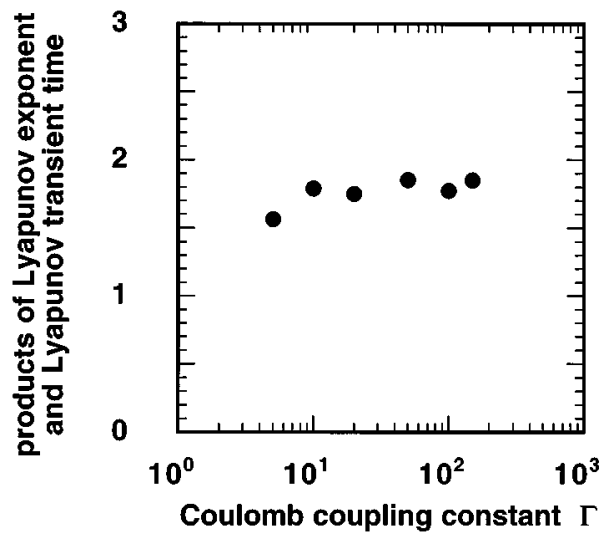

FIG. 7. Products of the Lyapunov exponent and the Lyapunov transient time for $\Gamma=1 \sim 150$. 


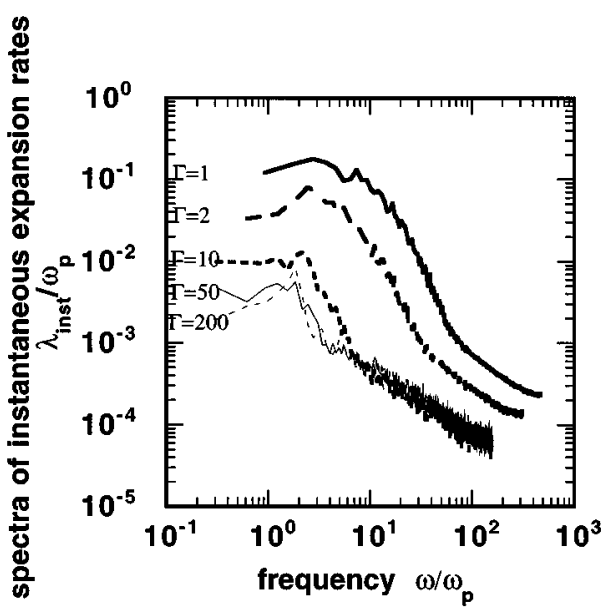

FIG. 8. Spectra of the instantaneous expansion rates for $\Gamma=1,2$, 10,50 , and 200.

\section{Lyapunov exponent}

The Lyapunov exponents are calculated for the various Coulomb coupling constants. With the rescaled method, we can get long-time data, 10000 time steps $\left(60 \omega_{p} t-200 \omega_{p} t\right)$, enough to regard the time-averaged instantaneous expansion rates as the asymptotic values. Figure 9 shows the dependence of the Lyapunov exponents on the Coulomb coupling constant. The Lyapunov exponents normalized by plasma frequency $\lambda / \omega_{p}$ vary from 0.83 to 0.12 and are proportional to $\Gamma^{-0.39}$ in the range $1 \leqslant \Gamma \leqslant 160$. The inverse of the plasma frequency $1 / \omega_{p}$ is the time that a particle with thermal speed takes to travel a Debye length $\lambda_{D}$. The Lyapunov exponents normalized by $v_{\mathrm{th}} / a, \lambda /\left(v_{\mathrm{th}} / a\right)$, vary from 1.44 to 2.05 and are proportional to $\Gamma^{-0.11}$ in the same range, where $a / v_{\text {th }}$ is the time that a particle with thermal speed takes to travel an ion sphere radius. The Lyapuhnov exponent normalized by $v_{\mathrm{th}} / a$ is almost independent of the Coulomb coupling constant and its magnitude is of the order of one. This result

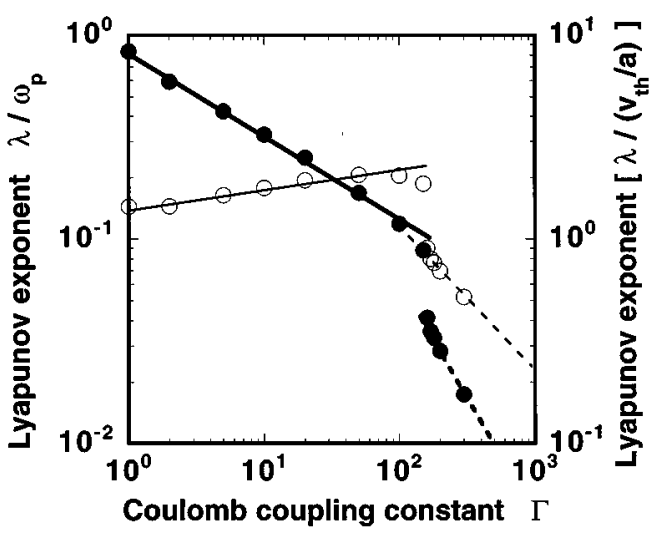

FIG. 9. Dependence of the Lyapunov exponents normalized by plasma frequency, $\lambda / \omega_{p}$, (closed circles) and those normalized by $v_{\mathrm{th}} / a, \lambda /\left(v_{\mathrm{th}} / a\right)$, (open circles) on the Coulomb coupling constant. Thick and thin solid lines are obtained by a least-squares method with the results of $\lambda / \omega_{p}$ and $\lambda /\left(v_{\mathrm{th}} / a\right)$, respectively, for $1 \leqslant \Gamma \leqslant 100$. Thick and thin dashed lines are obtained by a least-squares method with the result of $\lambda / \omega_{p}$ and $\lambda /\left(v_{\mathrm{th}} / a\right)$, respectively, for $170<\Gamma<300$. indicates that the trajectory instability in the phase space is scaled by the time $a / v_{\text {th }}$. As we will discuss later, this property may be universal for condensed matter in the liquid phase. The large jump of the Lyapunov exponent is observed near $\Gamma \sim 170$, which corresponds to the phase transition from the liquid to the solid state in the ion one-component plasma. This phase transition point is almost the same value that is calculated with the Monte Carlo simulations [10-13]. The Lyapunov exponents normalized by the plasma frequency decrease more rapidly from 0.04 and proportionally to $\Gamma^{-1.1}$ with the increase of the Coulomb coupling constant for $\Gamma>170$. This result indicates that the trajectory instability in the phase space occurs at a distance shorter than the ion sphere radius for solid plasmas.

In analogy to a rigid-body particle system, we may explain qualitatively the dependence of the Lyapunov exponent on the Coulomb coupling constant observed in the simulation. In a rigid-body particle system, the Lyapunov exponent is estimated from the mean relative velocity $V_{0}=4 / \sqrt{\pi} v_{\text {th }}$ the mean free path $l$, and an amplification factor of the displacement in a collision [3]. The amplification factor is found to be approximately $\ln [(l / 2 R)+1]$, where $R$ represents the radius of a rigid body particle. Since for a rigid body the density is $n=\left(4 \pi a^{3} / 3\right)^{-1}$ and the collision cross section is $\sigma=\pi R^{2}$, the mean free path is written as $4 a^{3} / 3 R^{2}$. In the rigid-body particle system, the Lyapunov exponent is then estimated as

$$
\lambda \sim \frac{V_{0}}{l} \ln \left[\frac{l}{2 R}+1\right]=3 \sqrt{\frac{1}{\pi}} \frac{v_{\mathrm{th}} R^{2}}{a^{3}} \ln \left[\frac{2}{3}\left(\frac{a}{R}\right)^{3}+1\right] .
$$

In the liquid plasma, the repulsive force between two particles is so large that a particle cannot get into the ion sphere of other particles and the nearest distance between two particles may be the same order as the ion sphere radius, independently of the Coulomb coupling constant. In the simulations, the closest distance increases slightly with the increase of the Coulomb coupling constant from $0.5 a$ to $0.9 a$ for $1 \leqslant \Gamma \leqslant 160$. Then the Lyapunov exponent normalized by $\left(v_{\mathrm{th}} / a\right)$ becomes of the order of one in the range 0.86-0.91 and becomes independent of the Coulomb coupling constant. The estimated value agrees roughly with the simulation result $\lambda /\left(v_{\mathrm{th}} / a\right) \sim 1.4 \Gamma^{0.11}$. The difference of the numerical factors between the simple estimation and the simulation results may be caused by neglecting many-body particle correlation in the estimation.

For the solid plasma, every particle is oscillating around a lattice point because it does not diffuse in the position coordinates space. The rigid-body particle model cannot be applied for the solid model, but the analogy of a nonlinear lattice system can be used. Then the trajectory instability can be caused by lattice vibration as a nonlinear lattice. In a harmonic lattice, the amplitude of lattice vibration is proportional to the square root of the kinetic energy of a particle and its oscillation period $2 \pi / \omega_{p}$ does not depend on its amplitude. If the nonlinearity of the lattice is small, it is expected that the growth rate of the trajectory instability is proportional to the square of the amplitude of the lattice vibration [4]. As a result, the Lyapunov exponent normalized by the plasma frequency is proportional to the kinetic en- 


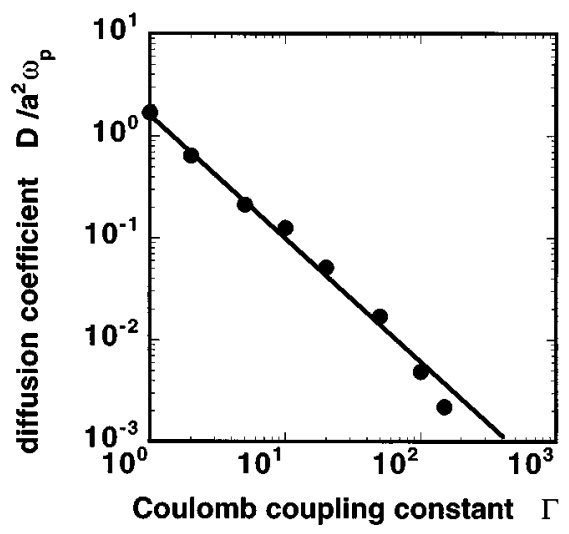

FIG. 10. Dependence of the normalized diffusion coefficients $D / a^{2} \omega_{p}$ on the Coulomb coupling constant. The solid line is obtained by a least-squares method with the results for $1 \leqslant \Gamma \leqslant 160$.

ergy, that is, $\Gamma^{-1}$. This estimation is also in good agreement with the simulation result $\lambda / \omega_{p} \sim \Gamma^{-1.1}$.

\section{Relation between the Lyapunov exponent and diffusion coefficient}

We conjecture that the Lyapunov exponents must be related to the macroscopic transport coefficients. To investigate this relation, the diffusion coefficients are calculated for the various Coulomb coupling constants. The diffusion coefficients are estimated from both the asymptotic mean-square deviations (MSD's) of particle position and the time integration of the velocity autocorrelation function. Both estimations agree quite well with each other for $1 \leqslant \Gamma \leqslant 160$.

Figure 10 shows the dependence of the diffusion coefficients on the Coulomb coupling constant. The normalized diffusion coefficients $D / a^{2} \omega_{p}$ are found to decrease from 2 to $2 \times 10^{-3}$ with the increase of the Coulomb coupling constant. The normalized diffusion coefficients are proportional to $\Gamma^{-1.18}$ for $1 \leqslant \Gamma \leqslant 160$. As shown in Fig. 11, the MSD of the particle position is proportional to time, that is, the diffusion coefficients are finite values in the same range. The phase transition point from the liquid to the solid state occurs near $\Gamma \sim 170$. Figure 11 also shows that the MSD of the par-

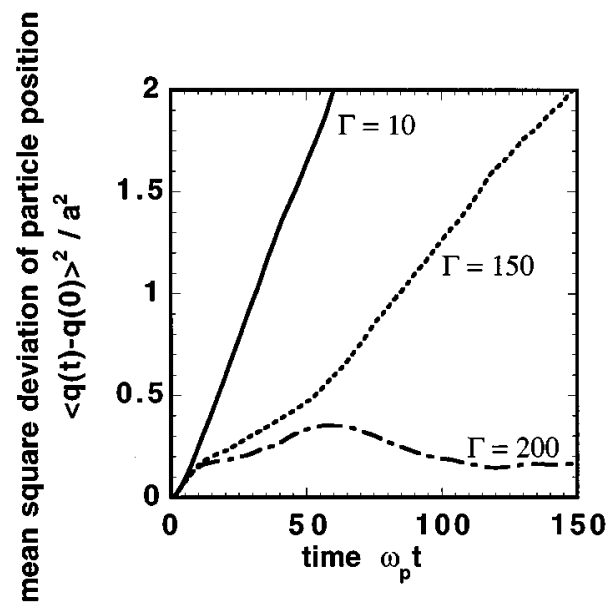

FIG. 11. Mean-square deviation of particle position as a function of time for $\Gamma=10,150$, and 200 .

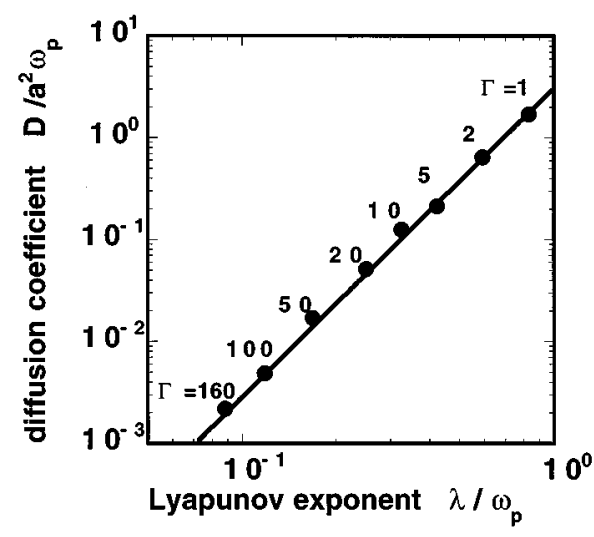

FIG. 12. Dependence of the normalized diffusion coefficient $D / a^{2} \omega_{p}$ on the Lyapunov exponent normalized by plasma frequency $\lambda / \omega_{p}$. The solid line is obtained by a least-squares method with the results for $1 \leqslant \Gamma \leqslant 160$.

ticle position is not proportional to time, but constant for $\Gamma=200$. The root-mean-square deviations of the particle position are $0.4 a-0.5 a$ in the range $170<\Gamma<300$. This result indicates that in the ion one-component solid plasmas, the amplitudes of the lattice vibration are $0.4 a-0.5 a$ for $170<\Gamma$ $<300$.

As shown in Fig. 12, the diffusion coefficient is proportional to the third power of the Lyapunov exponent [19] $D / a^{2} \omega_{p} \sim\left(\lambda / \omega_{p}\right)^{3}$ for the liquid state $1 \leqslant \Gamma \leqslant 160$. We find a relation between the Lyapunov exponents and the diffusion coefficients for ion one-component strongly coupled plasmas in the liquid state.

\section{CONCLUSION}

We have evaluated the Lyapunov exponent in the phase space for ion one-component plasmas with the use of the three-dimensional particle code SCOPE $[18,19]$. It has been observed with the nonrescaling method that the separation distance between two nearby trajectories diverges exponentially in time and that the deviation from the exponential growth occurs around the separations of $|\delta \mathbf{p}| \sim m v_{\text {th }}$ and $|\delta \mathbf{q}| \sim 3-4 \lambda_{D}$ for the various Coulomb coupling constants. In the transient stage, the initial displacement seeks out the phase-space eigendirection corresponding to the maximum Lyapunov exponent. After the Lyapunov transient time, the one-body distribution of the displacements relaxes to the isotropic normal distribution and the ratio $|\delta \mathbf{p}| /\left(|\delta \mathbf{q}| \omega_{p}\right)$ also relaxes to constants determined by the Coulomb coupling constant. It is also confirmed that the Lyapunov transient time is proportional to the inverse of the Lyapunov exponent.

The chaotic behavior of the instantaneous expansion rate is investigated. Bursts of the instantaneous expansion rate are observed for $\Gamma=1$, and even for $\Gamma>1$ the fluctuations of the instantaneous expansion rates are as large as their averages. These behaviors indicate that the trajectory instability is nonuniform in the phase space. The instantaneous expansion rates have broad spectra and consist of three different spectra. In order of frequency, the first component plateaus around the plasma frequency, the second decays as $f^{-2}$, and the last decays as $f^{-1}$.

The Lyapunov exponents normalized by plasma fre- 


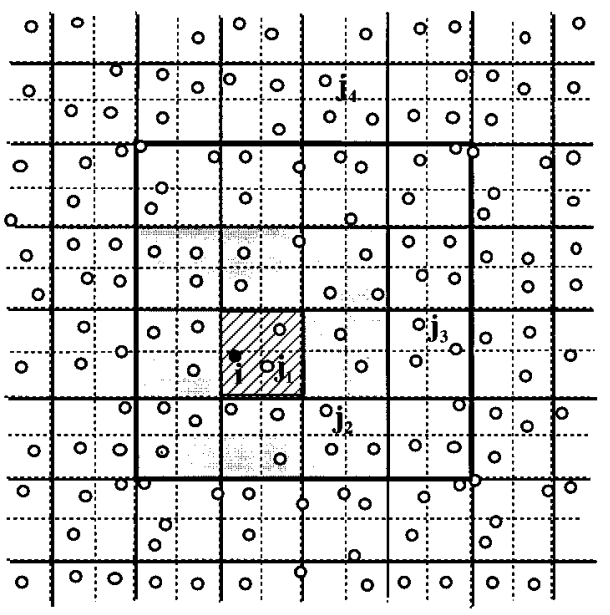

FIG. 13. Schematic diagram of two-dimensional meshes and particle positions with open and closed circles. Thin solid and dashed lines represent coarse grained (PP) and fine (PM) meshes, respectively. The thick solid line shows the two-dimensional unit system. Hatched and gray regions are called the PP region for the calculation of forces on a particle $i$ (closed circle).

quency are found to be proportional to $\Gamma^{-2 / 5}$ for $1 \leqslant \Gamma \leqslant 160$ and proportional to $\Gamma^{-6 / 5}$ for $\Gamma>170$. These states correspond to dense liquid and solid plasmas. The large jump of the Lyapunov exponent near $\Gamma \sim 170$ corresponds to the phase transition from the liquid to the solid state. The observed dependence is explained qualitatively in analogy to the rigid-body particle system and the weakly nonlinear lattice system for the liquid and the solid state, respectively. The diffusion coefficients are found to be proportional to the third power of the Lyapunov exponents in the liquid state.

\section{ACKNOWLEDGMENTS}

We thank Dr. M. Murakami, Dr. H. Sakagami, Dr. J. G. Wouchuk, Dr. S. Kato, and R. Ishizaki for useful comments. We also thank Professor K. Mima and Professor S. Nakai for their interest and encouragement. This work was partially supported by the Grant-in-Aid for Scientific Research from the Ministry of Education, Science, and Culture of Japan, Y. U. was supported by the Japan Society for the Promotion of Science for Japanese Junior Scientists.

\section{APPENDIX A}

In the PPPM method, three-dimensional meshes are introduced as shown by thin solid lines in Fig. 13, which illustrates two-dimensional meshes and particle positions by closed and open circles. We call here the thin solid and dashed meshes the PP mesh and the PM mesh. The role of the meshes is explained later. The thick solid line shows the two-dimensional unit system size. Every particle is booked in each corresponding PP mesh, namely, every particle has a "mesh address" and the booking is renewed at every time step. In the PPPM method, the mesh addressed is easily calculated by taking an integer of the particle position normalized by the mesh size. This is one of the advantage of the PPPM method.

In the PPPM method, the forces among all of the particles in the systems are calculated with two different methods for close particles (PP method) and distant particles (PM method). In the PP method, the direct particle-particle forces on the $i$ th particle (closed circle) are calculated by summing over the forces from the particles $j_{1}$ and $j_{2}$, which are located in the same PP mesh (hatched mesh) as the $i$ th particle and in its nearest-neighbor 26 PP meshes (gray meshes). This region of $27 \mathrm{PP}$ meshes is called the PP region. The forces from the particles $j_{3}$ and $j_{4}$ beyond the gray region are obtained using the PM method. The PM method is the same as the particle-in-cell (PIC) method [21] used in the ideal plasma particle simulation. Namely, the charge of a particle is assigned to grid points, and using charge density at the grid points, the finite-difference Poisson equation is solved to obtain the electric field of grid points. The force on the $i$ th particle is then interpolated from the electric field of the grid point.

The force on the $i$ th particle can be formally written as

$$
\begin{aligned}
\mathbf{F}_{i}= & e_{1} \sum_{j \in \mathrm{PP} \text { region }} \frac{e_{j}}{\left|\mathbf{q}_{i}-\mathbf{q}_{j}\right|^{3}}\left(\mathbf{q}_{i}-\mathbf{q}_{j}\right) \\
& +e_{i} \iiint_{\mathrm{PP} \text { region }} \frac{\rho_{e}}{\mid \mathbf{q}_{i}-\mathbf{q}^{3}}\left(\mathbf{q}_{i}-\mathbf{q}\right) d^{3} \mathbf{q} \\
& +e_{i}\left(\sum_{l, m, n}\left[\mathbf{E}_{l, m, n}\left(\mathbf{q}_{i}\right)-\mathbf{E}_{l, m, n}^{\prime}\left(\mathbf{q}_{i}\right)\right]\right)
\end{aligned}
$$

where $\mathbf{q}_{i}, \mathbf{q}_{j}$, and $\rho_{e}$ are the $i$ th and $j$ th particle positions and charge density of uniform background electrons, respectively. The first term expresses the direct particle-particle forces from particles in the PP region. The second term is the electric force from uniform background electrons in the PP region. The third term represents the force interpolated from the electric field of the grid points at the $i$ th particle position and $l, m, n$ are the three-dimensional grid numbers. $\mathbf{E}_{l, m, n}\left(\mathbf{q}_{i}\right)$ is the electric field (at the position $\mathbf{q}_{i}$ ) induced by all of particles in the system, while $\mathbf{E}_{l, m, n}^{\prime}\left(\mathbf{q}_{i}\right)$ is that induced by the particles in the PP region (hatched and gray meshes). $\mathbf{E}_{l, m, n}^{\prime}\left(\mathbf{q}_{i}\right)$ should be excluded from $\mathbf{E}_{l, m, n}\left(\mathbf{q}_{i}\right)$ because the Poisson equation is solved at once using the charge density at all grids to save computational cost. The detail will be discussed later.

In the PPPM method, errors of the force calculation arises from the PM method. We have made the following improvements [18] on the original PPPM method [20]: the introduction of fine meshes (the PM mesh) for the PM calculation in addition to the coarse-grained PP meshes, the use of the third-order spline function for both the charge assignment and the electric-field interpolation, the use of the fourth-order finite-difference scheme for the Poisson equation, and the exclusion of the double-counted electric field $\mathbf{E}_{l, m, n}^{\prime}\left(\mathbf{q}_{i}\right)$ in Eq. (A1).

A characteristic feature of the PIC method is the suppression of forces between close particles to simulate an ideal (collisionless) plasma. It causes the large error for the calculation of the forces among close particles. The absolute value of the error is determined by the distance between two particles normalized by the mesh size. It is shown [18] that by employing the third-order spline function and the fourthorder finite-difference scheme the error decays proportional 
to the inverse of the fourth power of the normalized distance between two particles, namely, $\sim\left(\left|\mathbf{q}_{i j}\right| / \Delta\right)^{-4}$, where $\Delta$ is the PM mesh size and $\mathbf{q}_{i j} \equiv \mathbf{q}_{i}-\mathbf{q}_{j}$. The fine mesh (PM mesh) is thus required for the PM calculation. On the contrary, the large size of the PP mesh (thin solid lines) is desirable to take many particles into account for the direct particleparticle force calculation. The size of the PP region can be chosen to be greater than the particle correlation length, which is approximately several ion sphere radii for strongly coupled plasmas. As shown in Fig. 13, the size of the fine mesh (dashed lines) is chosen to be half of the PP mesh in the present work. Then the normalized distance between nearest distant particles is greater than $2,\left|\mathbf{q}_{i j}\right| / \Delta>2$. By using this fine mesh size, the average error of the forces has been shown to be less than $0.02 \%$ [18] by direct comparison with the Ewald method [22], in which the forces among all particles are calculated with the periodic boundary condition. In the present work, the unit system is divided into $4^{3} \mathrm{PP}$ meshes, as shown in Fig. 13 and the number of particles is 500. The sizes of the PP mesh and the PP region are then approximately 3.2 and 9.6 times longer than the ion sphere radius, respectively. The direct particle-particle force is summed up over $500 \times\left(\frac{3}{4}\right)^{3}-(1 \approx 210)$ particles in average.

As described before, the electric field $\mathbf{E}_{l, m, n}^{\prime}\left(\mathbf{q}_{i}\right)$ induced by particles in the PP region should be subtracted from $\mathbf{E}_{l, m, n}\left(\mathbf{q}_{i}\right)$. In the PM method, using charge density at the grid points, the finite-difference Poisson equation is solved to obtain the electric field at grid points. In the fourth-order difference scheme, the charge density at the grid point $\left(l^{\prime}, m^{\prime}, n^{\prime}\right)$ induces the electric field of the grid point $(l, m, n)$,

$$
\begin{aligned}
\mathbf{E}(l, m, n)= & -i \frac{2 \pi \Delta \rho_{l^{\prime}, m^{\prime}, n^{\prime}}}{L^{\prime} M^{\prime} N^{\prime}} \\
& \times \sum_{k_{l}=0}^{L^{\prime}-1} \sum_{k_{m}=0}^{M^{\prime}-1} \sum_{k_{n}=0}^{N^{\prime}-1} \frac{\exp \left[i 2 \pi\left(\frac{k_{l}}{L^{\prime}}\left(l-l^{\prime}\right)+\frac{k_{m}}{M^{\prime}}\left(m-m^{\prime}\right)+\frac{k_{n}}{N^{\prime}}\left(n-n^{\prime}\right)\right)\right]}{\sin ^{2} \frac{\pi k_{1}}{L^{\prime}}\left(3+\sin ^{2} \frac{\pi k_{1}}{L^{\prime}}\right)+\sin ^{2} \frac{\pi k_{m}}{M^{\prime}}\left(3+\sin ^{2} \frac{\pi k_{m}}{M^{\prime}}\right)+\sin ^{2} \frac{\pi k_{n}}{N^{\prime}}\left(3+\sin ^{2} \frac{\pi k_{n}}{N^{\prime}}\right)} \\
& \times \frac{1}{3}\left\{\sin \frac{\pi k_{l}}{L^{\prime}} \cos \frac{\pi k_{l}}{L^{\prime}} \sin \frac{\pi k_{m}}{M^{\prime}} \cos \frac{\pi k_{m}}{M^{\prime}} \sin \frac{\pi k_{n}}{N^{\prime}} \cos \frac{\pi k_{n}}{N^{\prime}}\right\},
\end{aligned}
$$

where $\rho_{l^{\prime}, m^{\prime}, n^{\prime}}$ represents the charge density at the grid point $\left(l^{\prime}, m^{\prime}, n^{\prime}\right)$. Since the electric field $\mathbf{E}_{l, m, n}^{\prime}\left(\mathbf{q}_{i}\right)$ is induced by the charge density within the PP region, all of the charge densities in the PP region should be summed over. In principal, $L^{\prime}, M^{\prime}$, and $N^{\prime}$ should be infinite integers. Their numbers are chosen to be 256 times larger than the unit system size normalized by the PP mesh size since the contribution of the particles from a great distance on the electric field decays faster than the reciprocal of the third power of the distance.

\section{APPENDIX B}

As shown in Fig. 4, the normalized ratio of the magnitude of the momentum displacement to that of the position displacement becomes independent of the Coulomb coupling constant in the solid state. Let us consider a simple model to explain this ratio. The time derivative of the equation of motion, Eq. (6), is given by

$$
\delta \mathbf{p}=-\frac{d^{2} \Phi}{d \mathbf{q}^{2}} \delta \mathbf{q}-\frac{d^{2} \Phi}{d \mathbf{q}^{2}} \delta \mathbf{p} .
$$

The time derivative of the potential can be neglected for a small amplitude of lattice vibration. The lattice points can be given by $q=0, \pm d, \pm 2 d, \ldots$, where $d \sim 2 a$. The potential formed by the nearest-neighbor lattices can be expanded into the second order in the vibration amplitude near $q=0$ as

$$
\delta \mathbf{p}=-\frac{e^{2}}{d^{3}} \delta \mathbf{p}, \quad \delta \mathbf{q}=\delta \mathbf{p} .
$$

The ratio $|\delta \mathbf{p}| /|\delta \mathbf{q}|$ is then estimated as

$$
\frac{|\delta \mathbf{p}|}{|\delta \mathbf{q}|}=\sqrt{\frac{e^{2}}{m d^{3}}}=\omega_{p} \sqrt{\frac{1}{12}\left(\frac{2 a}{d}\right)^{3}} .
$$

The simulation results give that the ratio is of the order of $0.3 \omega_{p}$ for $\Gamma>170$. The ratio $a / d$ in Eq. (A3) is an invariant in the solid state. Assuming that $1.8 a<d<2 a$, the range of the ratio is $0.289 \omega_{p}<|\delta \mathbf{p}| / \mid \delta \mathbf{q}<0.390 \omega_{p}$. Thus this simple model shows that the ratio of $|\delta \mathbf{p}| /\left(|\delta \mathbf{q}| \omega_{p}\right)$ is a constant and is independent of the Coulomb coupling constant. If $d$ is defined as the nearest effective length between two particles in the liquid state, it decreases as the Coulomb coupling constant increases. This interpretation explains qualitatively the simulation result in the liquid state. 
[1] D. J. Evans, E. G. D. Cohen, and Gary P. Morriss, Phys. Rev. A 42, 5990 (1990).

[2] C. Amitrano and R. S. Berry, Phys. Rev. Lett. 68, 729 (1992).

[3] N. S. Krylov, Works on the Foundations of Statistical Physics (Princeton University Press, Princeton, 1979).

[4] M. Pettini and M. Landolfi, Phys. Rev. A 41, 768 (1990).

[5] S. Chandhuri, G. Gangopadhyay, and D. S. Ray, Phys. Rev. E 47, 311 (1993).

[6] P. Gaspard and G. Nicolis, Phys. Rev. Lett. 65, 1693 (1990).

[7] H. A. Posch and W. G. Hoover, Phys. Rev. A 38, 473 (1988).

[8] D. M. Barnett, The University of Texas at Austin, Institute of Fusion Studies Report No. IFSR 705, 1995 (unpublished).

[9] D. M. Barnett et al., Phys. Rev. Lett. 76, 1812 (1996).

[10] S. G. Brush, H. L. Sahlin, and E. Teller, J. Chem. Phys. 45, 2102 (1966).

[11] J. P. Hansen, Phys. Rev. A 8, 3096 (1973).

[12] S. Ichimaru et al., Statistical Physics of Dense Plasmas: Ther- modynamics, Transport Coefficients and Dynamic Correlations (North-Holland, Amsterdam, 1987).

[13] W. L. Slattery, G. D. Doolen, and H. E. DeWitt, Phys. Rev. A 26, 2255 (1982).

[14] A. J. Lichtenberg and M. A. Lieberman, Regular and Stochastic Motion (Springer-Verlag, New York, 1983).

[15] N. G. van Kampen, Stochastic Processes in Physics and Chemistry (North-Holland, Amsterdam, 1983).

[16] W. E. Wiesel, Phys. Rev. E 47, 3686 (1993).

[17] D. J. Evans, Phys. Rev. Lett. 69, 395 (1992).

[18] K. Nishihara, Kakuyugo Kenkyu 66, 253 (1991).

[19] H. Furukawa and K. Nishihara, Phys. Rev. A 42, 3532 (1990).

[20] R. W. Hockney and J. W. Eastwood, Computer Simulation using Particles (McGraw-Hill, New York, 1981).

[21] C. K. Birdsall and A. B. Langdon, Plasma Physics via Computer Simulation (McGraw-Hill, New York, 1995).

[22] P. P. Ewald, Ann. Phys. (N.Y.) 64, 253 (1921). 